\title{
Evaluation of Abicipar Pegol (an Anti-VEGF DARPin Therapeutic) in Patients With Neovascular Age-Related Macular Degeneration: Studies in Japan and the United States
}

\author{
Derek Kunimoto, MD, JD; Masahito Ohji, MD; Raj K. Maturi, MD; Tetsuju Sekiryu, MD; \\ Ying Wang, MD, PhD; Grace Pan, MS; Xiao-Yan Li, MD; Susan Schneider, MD; for the BAMBOO and \\ CYPRESS Study Groups
}

BACKGROUND AND OBJECTIVE: To evaluate comparability of abicipar pegol (abicipar) effects in patients with treatment-naïve neovascular age-related macular degeneration (nAMD) in Japan and the United States.

PATIENTS AND METHODS: Phase 2, multicenter, randomized, double-masked, 20-week studies (BAMBOO, Japan; CYPRESS, United States). Patients ( $\mathrm{n}=25$ each study) received three monthly intravitreal injections of abicipar $1 \mathrm{mg}$ or $2 \mathrm{mg}$ or five monthly intravitreal injections of ranibizumab $0.5 \mathrm{mg}$.

RESULTS: Mean best-corrected visual acuity change from baseline at week 16 (primary endpoint) for abicipar $1 \mathrm{mg}$, abicipar $2 \mathrm{mg}$, and ranibizumab was +7.8 letters, +8.9 letters, and +17.4 letters (BAMBOO); +4.4 letters, +10.1 letters, and +15.2 letters (CYPRESS). Mean central retinal thickness change from baseline was $-187.3 \mu \mathrm{m},-196.5 \mu \mathrm{m}$, and $-230.4 \mu \mathrm{m}$ (BAMBOO); -106.5 $\mu \mathrm{m},-112.8$ $\mu \mathrm{m}$, and $-124.4 \mu \mathrm{m}$ (CYPRESS). Uveitis or vitritis was reported in three abicipar-treated patients.

CONCLUSION: Abicipar demonstrated extended duration of effect and safety that were comparable between Japanese and non-Japanese patients with nAMD. Abicipar effectively treated Japanese patients with polypoidal choroidal vasculopathy.

[Ophthalmic Surg Lasers Imaging Retina. 2019;50:e10-e22.]

\section{INTRODUCTION}

Age-related macular degeneration (AMD), the leading cause of blindness in individuals 65 years of age and older in the developed world, is a progressive degeneration of the retinal pigment epithelium (RPE) and photoreceptors. ${ }^{1}$ Neovascular AMD (nAMD) is an advanced stage of the disease characterized by choroidal neovascularization (CNV). In nAMD, newly

\begin{abstract}
From the Retinal Consultants of Arizona, Phoenix (DK); Department of Ophthalmology, Shiga University of Medical Science, Shiga, Japan (MO); Midwest Eye Institute, Indianapolis, Indiana and Indiana University School of Medicine, Department of Ophthalmology, Indianapolis, Indiana (RKM); Department of Ophthalmology, Fukushima Medical University School of Medicine, Fukushima, Japan (TS); and Allergan plc, Irvine, California (YW, $\mathrm{GP}, \mathrm{X}-\mathrm{YL}, \mathrm{SS})$.
\end{abstract}

(c) 2019 Kunimoto, Ohji, Maturi, et al.; licensee SLACK Incorporated. This is an Open Access article distributed under the terms of the Creative Commons Attribution-NonCommercial 4.0 International (https://creativecommons.org/licenses/by-nc/4.0). This license allows users to copy and distribute, to remix, transform, and build upon the article non-commercially, provided the author is attributed and the new work is non-commercial. Originally submitted January 30, 2018. Revision received May 15, 2018. Accepted for publication May 29, 2018.

This study was presented in part at the Annual Meeting of the Association for Research in Vision and Ophthalmology (ARVO), May 7-11, 2017, Baltimore, MD.

Dr. Kunimoto is a consultant to Allergan, Genentech, and Graybug Vision. Dr. Ohji is a consultant for Alcon, Allergan, Bausch + Lomb, Bayer, Hoya, Novartis, Pfizer, and Santen and has received financial support for research from Alcon, Bayer, Novartis, Otsuka, Pfizer, Santen, and Senju. Dr. Maturi is a consultant for Allergan and Graybug Vision and has received clinical research funding from Allergan, Allegro Pharmaceuticals, Genentech, and Santen. Ms. Pan is a full-time employee of Allergan. Drs. Wang, Li, and Schneider are full-time employees of Allergan and have stock options. Dr. Sekiryu reports no relevant financial disclosures.

The authors thank the investigators and patients who participated in the BAMBOO and CYPRESS studies. They also acknowledge the research collaboration of Molecular Partners AG (Zurich, Switzerland) with Allergan in the clinical development of abicipar. Molecular Partners was responsible for preclinical and early clinical development of abicipar.

BAMBOO Study Group Principal Investigators: Tomohiro lida, Masashi Kakinoki, Hajime Kawamura, Ryusaburou Mori, Kunihiro Musashi, Yuji Oshima, Hiroshi Otake, Yoshihito Sakanishi, Tomoko Sawada, Tetsuju Sekiryu, Shinobu Takeuchi, Hiroko Terasaki. CYPRESS Study Group Principal Investigators: David Callanan, Thomas Chu, Sunil Gupta, Alexander Izad, Derek Kunimoto, Raj Maturi, Sunil Patel.

Address correspondence to Derek Kunimoto, MD, JD, 2152 S. Vineyard, Suite \#139, Bldg. 12, Mesa, AZ 85210; email: derek_kunimoto@yahoo.com. doi: 10.3928/23258160-20190129-13 
formed immature blood vessels originating in the choroid grow into the space beneath the neurosensory retina or RPE, and the vessel growth and exudation cause tissue disruption, subretinal disciform scar formation, and degeneration of the overlying neurosensory retina. ${ }^{1}$

Intravitreal injections of vascular endothelial growth factor (VEGF) blockers including ranibizumab (Lucentis; Genentech, South San Francisco, CA), bevacizumab (Avastin; Genentech, South San Francisco, CA), aflibercept (Eylea; Regeneron, Tarrytown, NY), and pegaptanib (Macugen; Bausch + Lomb, Rochester, NY), which can inhibit CNV lesion growth and exudation and stabilize or improve visual acuity (VA) in many patients, ${ }^{2}$ are currently the mainstay of treatment for nAMD. However, frequent injections are required for sustained efficacy. Data from large, prospective studies of ranibizumab administered monthly (ANCHOR ${ }^{3}$ and MARINA $^{4}$ ) or as needed (SUSTAIN ${ }^{5}$ and HORIZON ${ }^{6}$ ) in patients with nAMD have suggested that outcomes are better with intensive, monthly dosing of ranibizumab. Moreover, subtypes of nAMD that have been identified (typical nAMD, polypoidal choroidal vasculopathy [PCV], and retinal angiomatous proliferation) appear to differ in their responsiveness to anti-VEGF treatment. ${ }^{7-9}$ In a cross-sectional, retrospective study, most patients diagnosed with nAMD who demonstrated a poor response to ranibizumab had atypical nAMD, with the majority having PCV. ${ }^{10}$ These results suggest a need for novel treatments for nAMD that require less-frequent administration (decreasing the burden of treatment) and that are effective in atypical subtypes of nAMD lesions.

Abicipar pegol (abicipar; Allergan, Dublin, Ireland), a member of the family of DARPin therapeutics (Molecular Partners, Zurich, Switzerland), is a pegylated $34 \mathrm{kDa}$ recombinant ankyrin repeat protein that binds all isoforms of VEGF-A with high affinity and specificity. ${ }^{11-13}$ Abicipar binds to VEGF- ${ }_{165}$ with higher affinity than ranibizumab (Kd of $2 \mathrm{pM}^{13}$ versus $46 \mathrm{pM}^{14}$ ) and has demonstrated potent inhibition of anti-VEGF activity (IC50 of $17 \mathrm{pM}$ ) in a spheroid sprouting assay. ${ }^{11}$ Studies of aqueous humor concentrations of anti-VEGF agents after intravitreal administration in human subjects have reported a longer intraocular half-life for abicipar ( $\geq 13$ days) compared with 7.2 days for ranibizumab, ${ }^{15}$ with abicipar present at concentrations orders of magnitude above the IC50 for VEGF inhibition for at least 12 weeks after intravitreal administration of a $0.4-\mathrm{mg}$ dose. ${ }^{11}$

These findings suggest that abicipar may demonstrate a longer duration of effect compared with ranibizumab, and that the required frequency of in- travitreal injections may be markedly reduced. Abicipar is currently under evaluation as a long-acting intravitreal anti-VEGF therapy for nAMD. In stage 3 of the phase 2 REACH study (NCT01397409), abicipar $1 \mathrm{mg}$ and $2 \mathrm{mg}$ improved best-corrected VA (BCVA) and central retinal thickness (CRT; retinal thickness in the $1 \mathrm{~mm}$ diameter central subfield) similarly to ranibizumab $0.5 \mathrm{mg}$, but with a reduced number of injections. ${ }^{16}$

In this report, we present the results of two additional small, exploratory, phase 2 clinical studies (BAMBOO; CYPRESS) assessing the safety and efficacy of abicipar $1 \mathrm{mg}$ and $2 \mathrm{mg}$ in Japan and the United States, respectively. Ranibizumab $0.5 \mathrm{mg}$ was also used in these studies as an active control. Our study objective was to evaluate the comparability of abicipar effects in Japanese and non-Japanese patients with nAMD. Accordingly, the clinical studies were nearly identical in design, such that regulatory requirements of the Pharmaceuticals and Medical Device Agency in Japan could be met.

\section{PATIENTS AND METHODS}

Two phase 2, multicenter, randomized, doublemasked clinical studies evaluated the safety and treatment effects of abicipar $1 \mathrm{mg}$, abicipar $2 \mathrm{mg}$, and ranibizumab $0.5 \mathrm{mg}$ in treatment-naïve patients with nAMD in Japan (BAMBOO study) and the United States (CYPRESS study). The studies were conducted from September 2014 to December 2015 (BAMBOO) and July 2014 to March 2015 (CYPRESS) in accordance with the International Council for Harmonization Good Clinical Practice Guideline. The study protocol was approved by an institutional review board at each site, and all patients provided written informed consent.

\section{Patient Selection}

Patient inclusion criteria included active subfoveal and/or juxtafoveal CNV secondary to AMD in the study eye, with retinal fluid and/or leakage affecting the fovea, diagnosed within the 12 months before screening. Active CNV was determined by the presence of retinal fluid on spectral-domain optical coherence tomography (SD-OCT) as confirmed by the central reading center (University of Wisconsin Fundus Photography Reading Center, Madison, Wisconsin), with CRT in the study eye greater than $320 \mu \mathrm{m}$ on the Spectralis machine (Heidelberg Engineering, Heidelberg, Germany) or greater than 300 $\mu \mathrm{m}$ on the Cirrus machine (Carl Zeiss Meditec, Jena, Germany). Other key inclusion criteria included CNV lesion area greater than $50 \%$ of the total lesion area and not larger than 12 disc areas on fluorescein angi- 


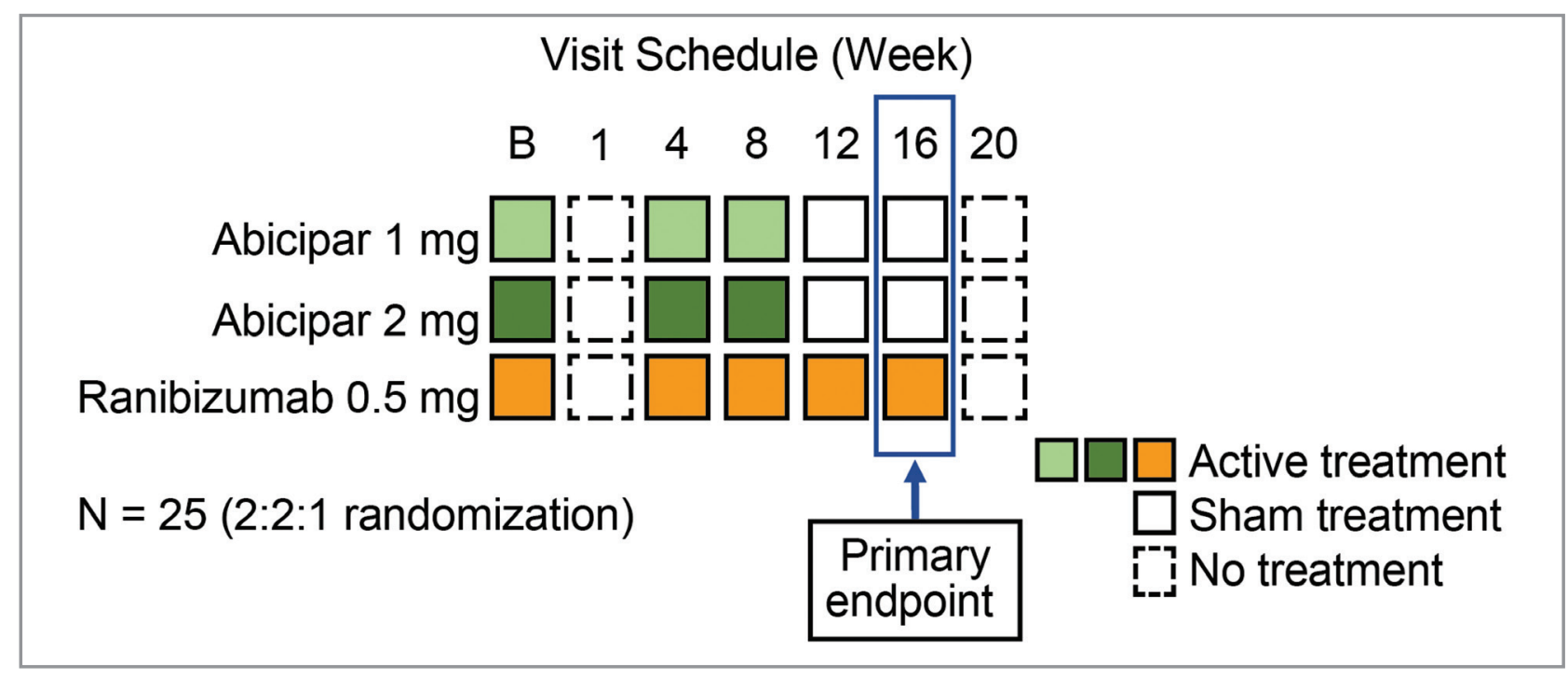

Figure 1. Study design schematic. Additional safety study visits were scheduled 2 days after the baseline and week 8 visits. B = baseline.

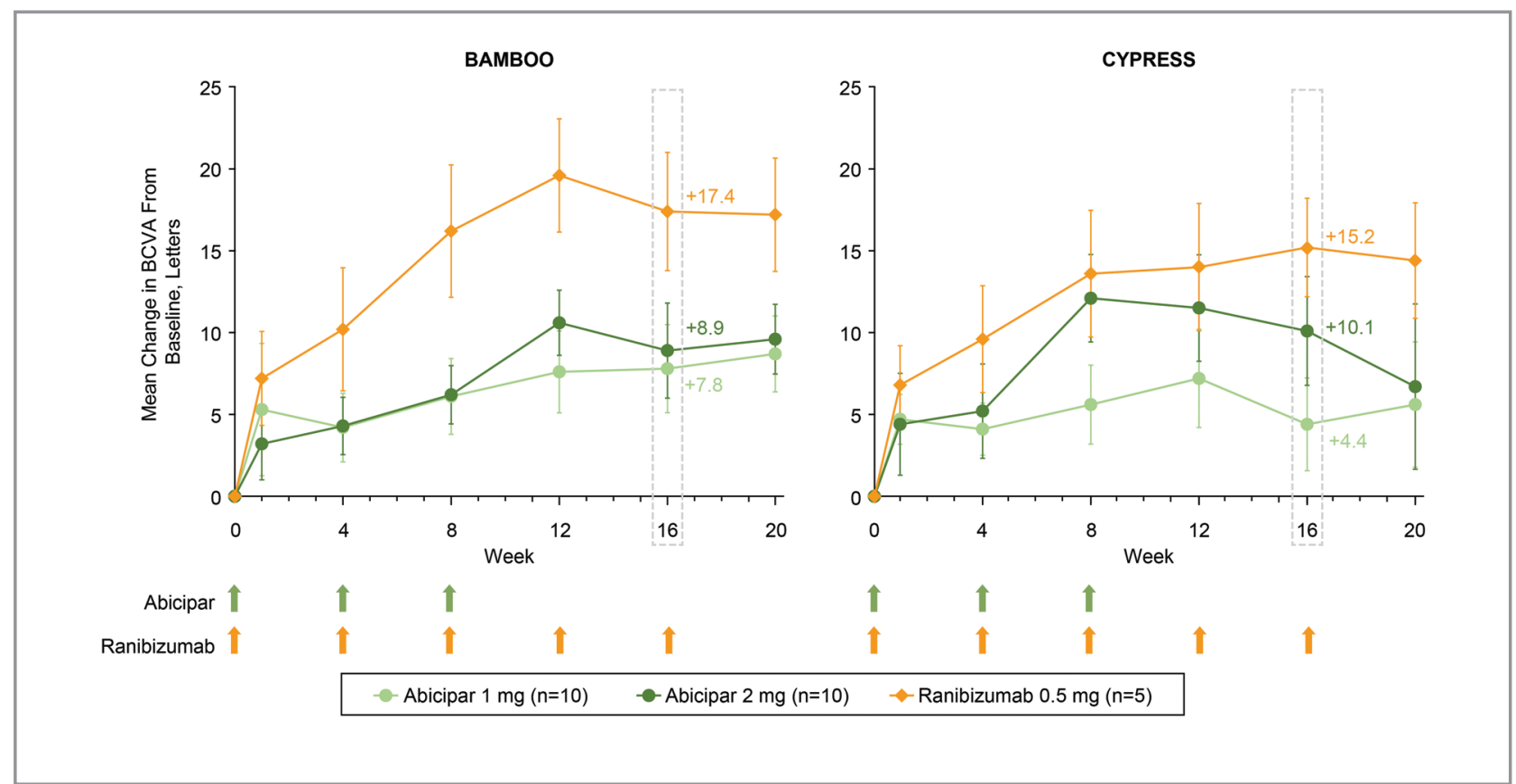

Figure 2. Mean change from baseline in best-corrected visual acuity (BCVA) at each visit in the BAMBOO and CYPRESS studies. The primary endpoint was at week 16 . Green and orange arrows indicate treatment with abicipar and ranibizumab, respectively. Missing data were imputed with the last observation carried forward. Errors bars indicate standard error of the mean.

ography in the study eye, and BCVA between 24 and 75 Early Treatment Diabetic Retinopathy Study (ETDRS) letters (approximately 20/32 to 20/320 Snellen equivalent) in the study eye and 34 or more ETDRS letters (approximately 20/200 or better) in the fellow eye. Patients were required to be at least 50 years of age, and those in the BAMBOO study were required to be of Japanese descent. Inclusion was limited to patients who had not received any previously ad- ministered therapy (verteporfin [Visudyne; Bausch + Lomb, Rochester, NY] or anti-angiogenic therapy, approved or investigational) for nAMD in the study eye. The BAMBOO study included indocyanine green angiography (ICGA) at screening because of the high prevalence of PCV in Japanese patients with nAMD. ${ }^{17}$ Patients with or without PCV could be included in the study.

Exclusion criteria for study eyes included presence 


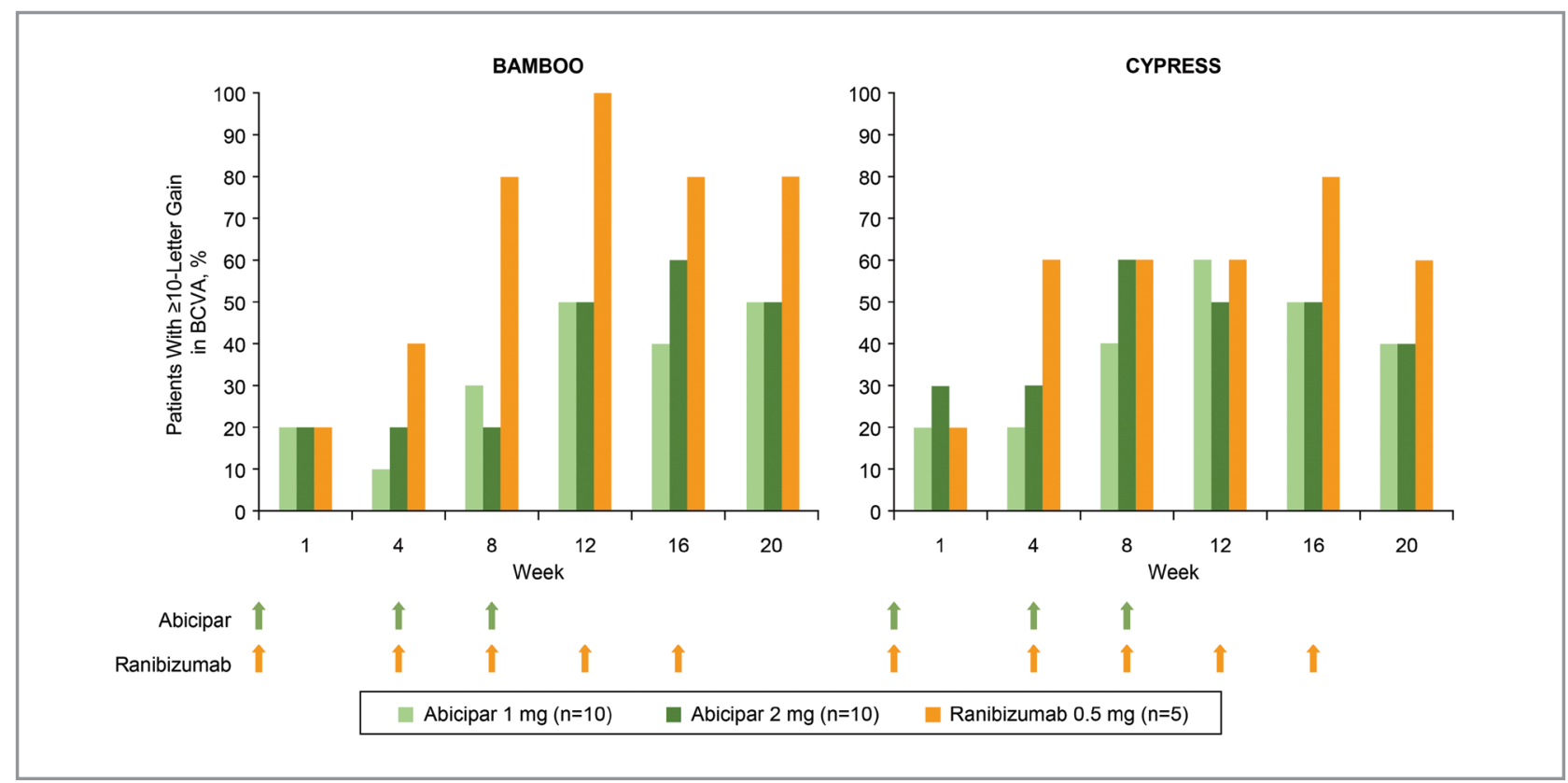

Figure 3. Percentage of patients who gained at least 10 letters in best-corrected visual acuity (BCVA) from baseline at each visit in the BAMBOO and CYPRESS studies. Green and orange arrows indicate treatment with abicipar and ranibizumab, respectively. Missing data were imputed with the last observation carried forward.

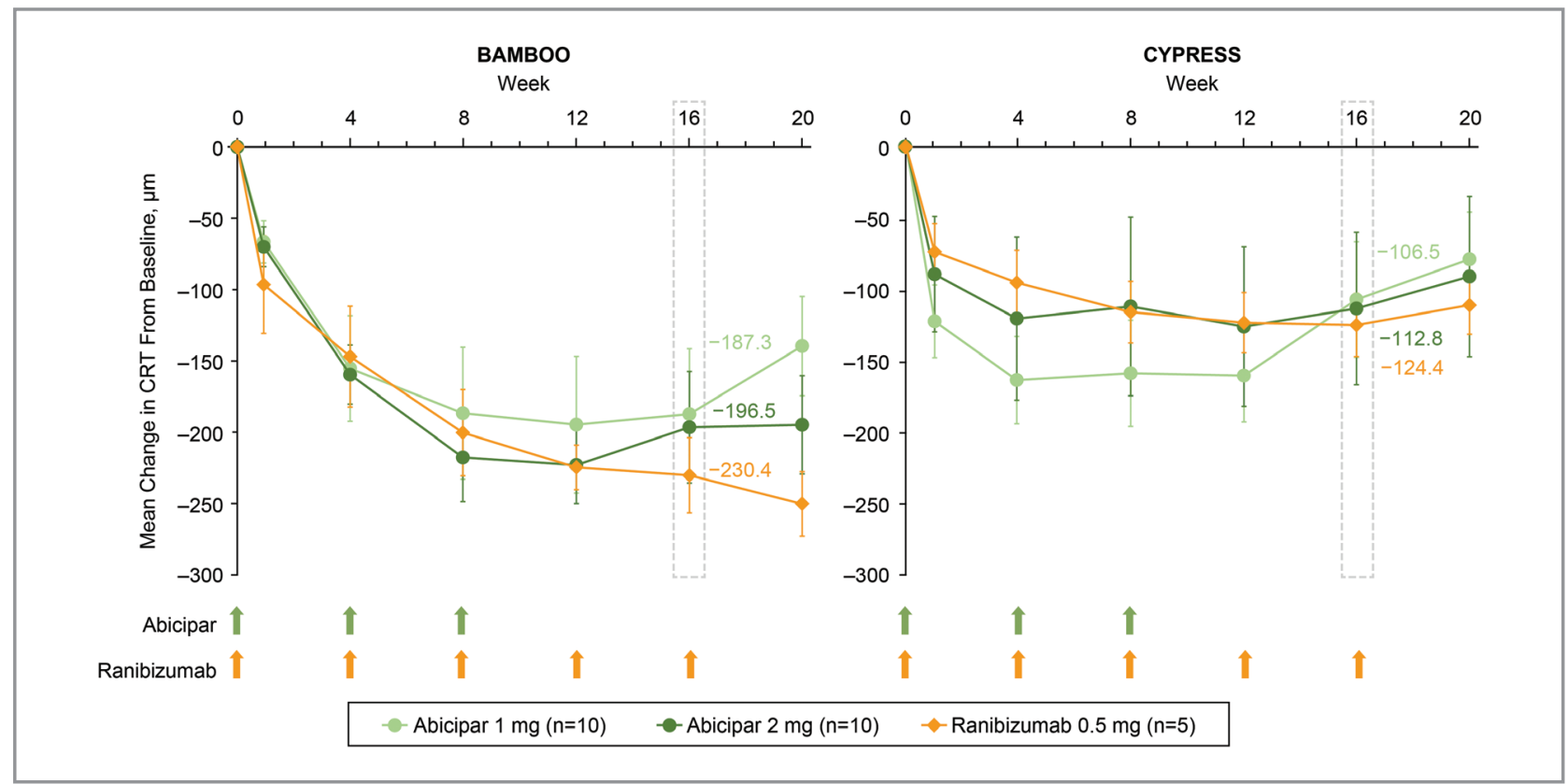

Figure 4. Mean change from baseline in central retinal thickness (CRT) at each visit in the BAMBOO and CYPRESS studies. Green and orange arrows indicate treatment with abicipar and ranibizumab, respectively. Missing data were imputed with the last observation carried forward. Errors bars indicate standard error of the mean.

of a retinal angiomatous proliferation lesion, structural damage to the center of the macula likely to preclude improvement in BCVA (eg, subretinal fibrosis/ scarring), vitreous hemorrhage, macular hemorrhage greater than $50 \%$ of the lesion area or greater than 1 disc area in size involving the center of fovea, history of vitrectomy or submacular surgery, history of treatment with fluocinolone acetonide implant within the past 36 months or with other ocular corticosteroid injections within the past 6 months, and high myopia 


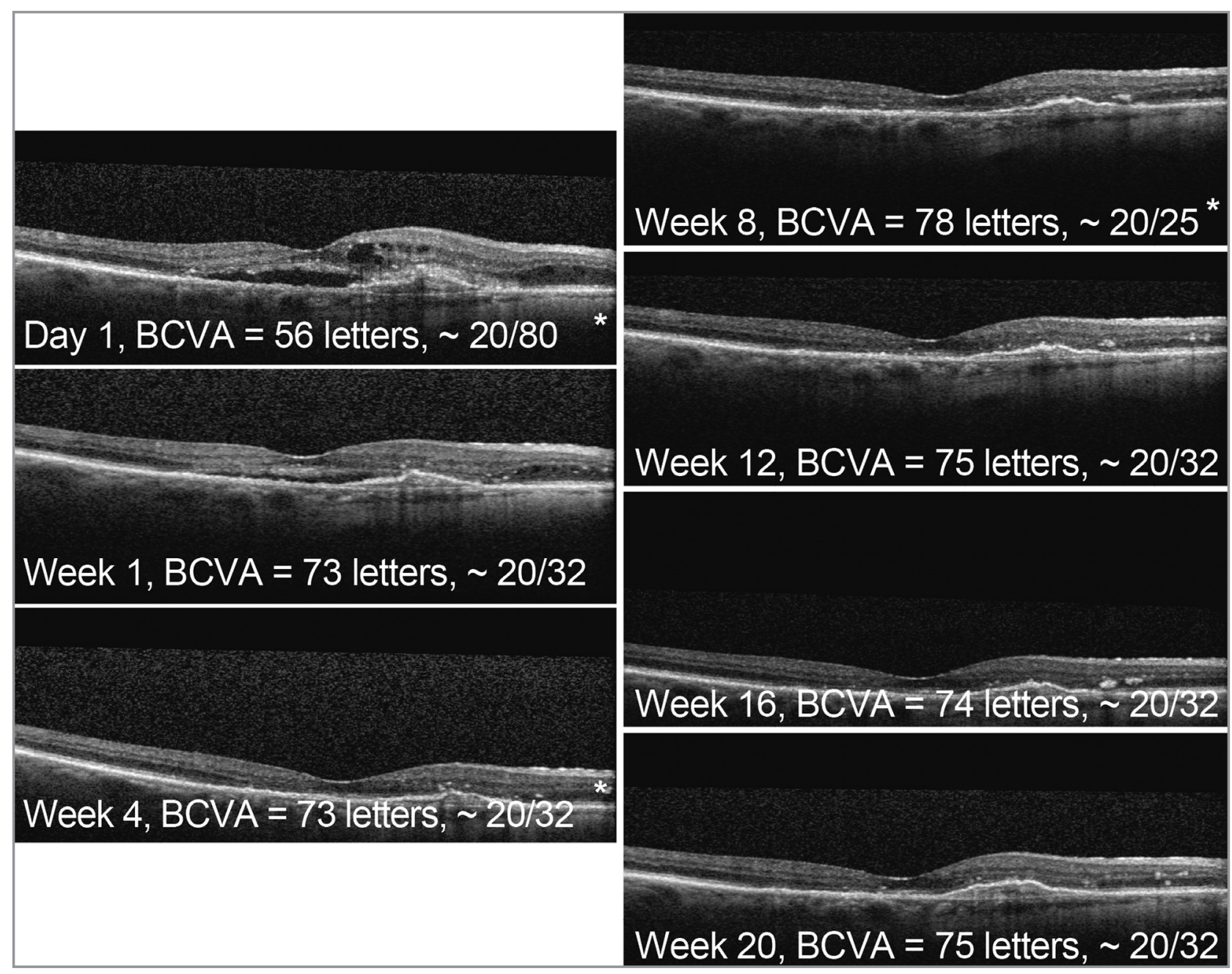

Figure 5. Spectral-domain optical coherence tomography images and best-corrected visual acuity (BCVA) in a 91-year-old Caucasian female with neovascular age-related macular degeneration in the right eye treated with abicipar $2 \mathrm{mg}$ in the CYPRESS study. Asterisk indicates visit with abicipar intravitreal injection (baseline, week 4 , and week 8).

( $\leq-6$ diopters spherical equivalent). Other key exclusion criteria included history of recurrent or active systemic or ocular / intraocular infection or inflammation, and any systemic or ophthalmic medical condition that in the opinion of the investigator could prevent safe participation in the study and adherence to the study visits, or that could confound the study results.

If both eyes met the eligibility criteria, the eye with the worse BCVA was selected as the study eye.

\section{Randomization and Study Treatment}

Each study included a screening visit (day -21 to day -2 ); a baseline visit (day 1); and visits at weeks $1,4,8,12,16$, and 20 . In addition, patients were seen at visits 2 days after the baseline and week 8 visits for pharmacokinetic blood sampling and safety assessments only. At the baseline visit, 25 patients in each study were randomly assigned in a 2:2:1 ratio to treatment with abicipar $1 \mathrm{mg}(\mathrm{n}=10)$, abicipar 2 $\mathrm{mg}(\mathrm{n}=10)$, and ranibizumab $0.5 \mathrm{mg}(\mathrm{n}=5)$ in the study eye. Ranibizumab was administered by intravitreal injection of $50 \mu \mathrm{L}$ of a $10 \mathrm{mg} / \mathrm{mL}$ solution at baseline and every 4 weeks through week 16 (a total of five monthly injections). In the abicipar treatment arms, abicipar was administered by intravitreal injection of $50 \mu \mathrm{L}$ of a $20 \mathrm{mg} / \mathrm{mL}$ or $40 \mathrm{mg} / \mathrm{mL}$ solution at baseline, week 4, and week 8 (a total of three monthly injections), and a sham procedure was performed at weeks 12 and 16 to maintain masking (Figure 1). Patients, the investigator responsible for safety assessments (except the post-injection assessment), and all site personnel involved in efficacy assessments were masked to patients' treatment assignments. The ophthalmologist who performed the study drug injections was unmasked to the treatment. 


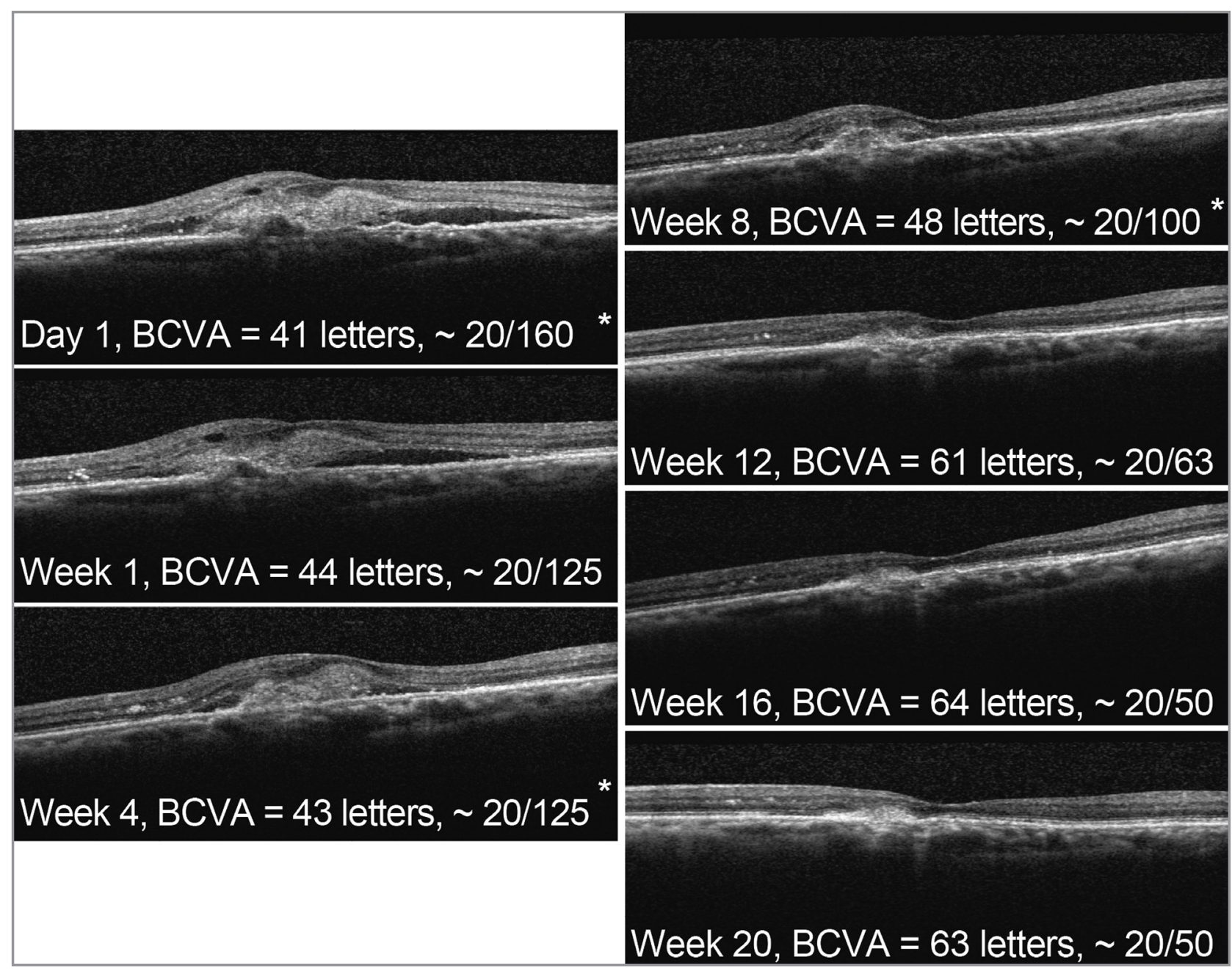

Figure 6. Spectral-domain optical coherence tomography images and best-corrected visual acuity (BCVA) in a 77-year-old Japanese male with neovascular age-related macular degeneration and polypoidal choroidal vasculopathy in the left eye treated with abicipar $2 \mathrm{mg}$ in the BAMBOO study. Asterisk indicates visit with abicipar intravitreal injection (baseline, week 4, and week 8).

Study treatment was to be suspended if the study eye developed treatment-related severe intraocular inflammation, but it could be reinitiated at the investigator's discretion after the inflammation resolved. Escape to standard-of-care (SoC) after the week 12 visit was allowed if there was a 15-letter or greater decrease in BCVA compared with the worst BCVA at previous visits, attributable to a lack of treatment effect. Patients who escaped to SoC were to be followed for safety assessments through week 20 .

\section{Assessments and Outcome Measures}

BCVA and CRT were assessed at baseline and at weeks $1,4,8,12,16$, and 20 . The primary efficacy measure was BCVA evaluated using a modification of the ETDRS method ${ }^{18}$ by certified, masked evaluators and expressed as the number of letters read. In the BAMBOO study, the ETDRS eye chart used Arabic numerals rather than Roman letters, as these are easier for Japanese patients to read. The secondary efficacy measure was CRT evaluated using SD-OCT. SDOCT images were evaluated and CRT was quantified by masked readers at the central reading center. Safety measures included treatment-emergent adverse events (AEs) (ie, AEs with onset after the initiation of treatment, or ongoing AEs that increased in severity after the onset of treatment), BCVA, slit-lamp biomicroscopy, ophthalmoscopy, and intraocular pressure.

The primary endpoint was mean change from baseline in BCVA at week 16. Secondary endpoints included mean change from baseline BCVA at week 20 , proportion of patients with a 10-letter or greater gain in BCVA from baseline, proportion of patients with a 15-letter or greater gain in BCVA from baseline, and mean change in CRT from baseline. In addition, the proportion of patients with stable vision 
TABLE 1

Baseline Patient and Lesion Characteristics

\begin{tabular}{|c|c|c|c|c|c|c|}
\hline \multirow[b]{2}{*}{ Characteristic } & \multicolumn{3}{|c|}{ BAMBOO (Japan) } & \multicolumn{3}{|c|}{ CYPRESS (United States) } \\
\hline & $\begin{array}{l}\text { Abicipar } 1 \mathrm{mg} \\
(\mathrm{n}=10)\end{array}$ & $\begin{array}{l}\text { Abicipar } 2 \mathrm{mg} \\
(\mathrm{n}=10)\end{array}$ & $\begin{array}{l}\text { RAN } 0.5 \mathrm{mg} \\
(\mathrm{n}=5)\end{array}$ & $\begin{array}{l}\text { Abicipar } 1 \mathrm{mg} \\
(\mathrm{n}=10)\end{array}$ & $\begin{array}{l}\text { Abicipar } 2 \mathrm{mg} \\
(\mathrm{n}=10)\end{array}$ & $\begin{array}{l}\text { RAN } 0.5 \mathrm{mg} \\
(\mathrm{n}=5)\end{array}$ \\
\hline Mean age, years & 75.6 & 71.8 & 76.6 & 81.0 & 85.5 & 83.8 \\
\hline Range & $64-68$ & $63-81$ & $65-89$ & $66-91$ & $71-95$ & 74-92 \\
\hline Female, n (\%) & $1(10)$ & $2(20)$ & $1(20)$ & $9(90)$ & $7(70)$ & $4(80)$ \\
\hline \multicolumn{7}{|l|}{ Race/ethnicity, n (\%) } \\
\hline Japanese & $10(100)$ & $10(100)$ & $5(100)$ & $0(0)$ & $0(0)$ & $0(0)$ \\
\hline Caucasian & $0(0)$ & $0(0)$ & $0(0)$ & $10(100)$ & $10(100)$ & $5(100)$ \\
\hline Mean BCVA, letters & 54.3 & 58.5 & 55.8 & 55.2 & 59.0 & 57.6 \\
\hline Range & $24-72$ & $27-75$ & $47-70$ & $33-70$ & $42-74$ & $30-72$ \\
\hline $\begin{array}{l}\text { BCVA > } 70 \text { letters } \\
\text { (20/40 Snellen } \\
\text { equivalent), } n(\%)\end{array}$ & $3(30)$ & $4(40)$ & $0(0)$ & $0(0)$ & $2(20)$ & $2(40)$ \\
\hline Mean CRT, $\mu \mathrm{m}$ & 475.1 & 438.7 & 470.0 & 443.8 & 383.8 & 348.8 \\
\hline Range & $296-840$ & 288-591 & $363-538$ & $285-643$ & $278-792$ & 313-395 \\
\hline \multicolumn{7}{|l|}{ Lesion type, n (\%) } \\
\hline \multicolumn{7}{|l|}{ CNV $>50 \%$} \\
\hline $\begin{array}{l}\text { Predominantly } \\
\text { classic }\end{array}$ & $3(30)$ & $5(50)$ & $2(40)$ & $5(50)$ & $3(30)$ & $1(20)$ \\
\hline Minimally classic & $1(10)$ & $2(20)$ & $3(60)$ & $1(10)$ & $1(10)$ & $0(0)$ \\
\hline Occult & $6(60)$ & $3(30)$ & $0(0)$ & $4(40)$ & $5(50)$ & $3(60)$ \\
\hline CNV $<50 \%$ & $0(0)$ & $0(0)$ & $0(0)$ & $0(0)$ & $1(10)$ & $1(20)$ \\
\hline $\begin{array}{l}\text { PCV component, } \\
\text { n (\%) }\end{array}$ & $5(50)$ & $6(60)$ & $3(60)$ & NA & NA & NA \\
\hline
\end{tabular}

$B C V A=$ best-corrected visual acuity measured with the Early Treatment Diabetic Retinopathy Study method; $C N V=$ choroidal neovascularization; $C R T=$ central retinal thickness; $N A=$ not available; $P C V=$ polypoidal choroidal vasculopathy determined by indocyanine green angiography; $R A N=$ ranibizumab

(defined as < 15-letter loss in BCVA from baseline) was evaluated at each visit.

\section{Statistical Analysis}

Efficacy outcomes in each treatment arm were summarized for all scheduled visits using descriptive statistics in the modified intent-to-treat population of all randomized and treated patients with at least one follow-up visit. Subgroup descriptive statistics of efficacy parameters in the BAMBOO study were similarly performed in patients after dividing them by the presence or absence of PCV at baseline. For all analyses, missing values were imputed using the last-observation-carried-forward method.

No statistical analysis comparing outcomes between treatment arms was planned. The planned sample size was 25 patients for each study (10 pa- tients in both the abicipar $1 \mathrm{mg}$ and $2 \mathrm{mg}$ groups and five patients in the ranibizumab group). The sample size was determined empirically and in collaboration with the Pharmaceuticals and Medical Device Agency in Japan. Based on preliminary findings of BCVA and CRT changes in patients treated with abicipar, it was decided that a sample size of 10 patients in the abicipar $1 \mathrm{mg}$ and $2 \mathrm{mg}$ arms in each study would provide adequate data for evaluation of the comparability of abicipar effects in Japanese and non-Japanese patients.

\section{RESULTS}

A total of 25 treatment-naïve patients with nAMD were enrolled in both the BAMBOO and CYPRESS studies. Table 1 lists baseline demographics in the studies. Age, gender, and race / ethnicity were similar across treatment arms within each study. The Japa- 
TABLE 2

Efficacy Outcomes in Japanese Patients With or Without PCV (BAMBOO Study)

\begin{tabular}{|c|c|c|c|c|c|c|}
\hline \multirow[b]{2}{*}{$\begin{array}{l}\text { Outcome } \\
\text { Measure }\end{array}$} & \multicolumn{3}{|c|}{ Patients With PCV* } & \multicolumn{3}{|c|}{ Patients Without PCV† } \\
\hline & $\begin{array}{l}\text { Abicipar } 1 \mathrm{mg} \\
(\mathrm{n}=5)\end{array}$ & $\begin{array}{l}\text { Abicipar } 2 \mathrm{mg} \\
(\mathrm{n}=6)\end{array}$ & $\begin{array}{l}\text { RAN } 0.5 \mathrm{mg} \\
(\mathrm{n}=3)\end{array}$ & $\begin{array}{l}\text { Abicipar } 1 \mathrm{mg} \\
(\mathrm{n}=5)\end{array}$ & $\begin{array}{l}\text { Abicipar } 2 \mathrm{mg} \\
(\mathrm{n}=4)\end{array}$ & $\begin{array}{l}\text { RAN } 0.5 \mathrm{mg} \\
(\mathrm{n}=2)\end{array}$ \\
\hline $\begin{array}{l}\text { Mean (SEM) } \\
\text { change from } \\
\text { baseline } \\
\text { BCVA at week } \\
\text { 16, letters }\end{array}$ & $+6.0(4.4)$ & $+9.8(4.6)$ & $+12.3(3.2)$ & $+9.6(3.4)$ & $+7.5(3.1)$ & $+25.0(2.0)$ \\
\hline $\begin{array}{l}\text { Mean (SEM) } \\
\text { change from } \\
\text { baseline CRT } \\
\text { at week 16, } \\
\mu \mathrm{m}\end{array}$ & $-268.6(69.6)$ & $-191.2(64.1)$ & $-253.7(10.1)$ & $-106.0(37.4)$ & $-204.5(35.5)$ & $-195.5(68.5)$ \\
\hline
\end{tabular}

Missing data were imputed with the last observation carried forward.

* Determination of definite polyps in the study eye at screening.

tDetermination of absent or questionable polyps in the study eye at screening.

$B C V A=$ best-corrected visual acuity; $C R T=$ central retinal thickness; $P C V=$ polypoidal choroidal vasculopathy; SEM = standard error of the mean

nese patient population in BAMBOO was younger (mean of 74.3 years; range: 63 years to 89 years) than the non-Japanese patient population in CYPRESS (mean of 83.4 years; range: 66 years to 95 years), and $84 \%$ of the Japanese patients were male, whereas $80 \%$ of the non-Japanese patients were female. In both studies, at least $50 \%$ of patients in each treatment arm had minimally classic or occult lesions in the study eye (Table 1).

All patients in each treatment arm completed the CYPRESS study. In the BAMBOO study, 22 of 25 patients completed the study. Reasons for early discontinuation in BAMBOO were mild iritis/vitritis (one of 10 patients in the abicipar $1 \mathrm{mg}$ arm), mild iritis (one of 10 patients in the abicipar $2 \mathrm{mg}$ arm), and acute angle-closure glaucoma (one of five patients in the ranibizumab arm). No patients in either study escaped to SoC treatment prior to the week 20 assessments.

\section{Visual Outcomes}

In both studies, analysis of mean change from baseline in BCVA showed improvement in BCVA in each treatment arm at all follow-up visits (Figure 2). Mean (standard error of the mean [SEM]) change from baseline in BCVA at week 16 (primary endpoint, 8 weeks after the last abicipar injection and 4 weeks after the last ranibizumab injection) was +7.8 (2.7) letters, +8.9 (2.9) letters, and +17.4 (3.6) letters in the BAMBOO study and +4.4 (2.8) letters, +10.1 (3.3) letters, and +15.2 (3.0) letters in the CYPRESS study in the abicipar 1 $\mathrm{mg}$, abicipar $2 \mathrm{mg}$, and ranibizumab arms, respectively (Figure 2). Results of the analysis of patients with 10 letters or 15 letters gain must be interpreted with caution because of a possible ceiling effect, since the baseline BCVA in patients ranged up to 75 letters (Table 1). Nonetheless, the percentage of patients who gained at least 10 letters in BCVA from baseline was similar in the $1 \mathrm{mg}$ and $2 \mathrm{mg}$ abicipar arms and consistent across studies (eg, five of 10 [50\%] with abicipar $1 \mathrm{mg}$ and 2 $\mathrm{mg}$ in BAMBOO and four of 10 [40\%] with abicipar $1 \mathrm{mg}$ and $2 \mathrm{mg}$ in CYPRESS at week 20; Figure 3). At week 20, 12 weeks after the last abicipar injection and 4 weeks after the last ranibizumab injection, five of 10 ( $50 \%$ ), five of $10(50 \%)$, and four of five $(80 \%)$ patients (BAMBOO) and four of $10(40 \%)$, four of $10(40 \%)$, and three of five $(60 \%)$ patients (CYPRESS) had gained at least 10 letters in BCVA from baseline in the abicipar 1 $\mathrm{mg}$, abicipar $2 \mathrm{mg}$, and ranibizumab arms, respectively. At week 20, 12 weeks after the last abicipar injection and 4 weeks after the last ranibizumab injection, three of $10(30 \%)$, two of $10(20 \%)$, and three of five $(60 \%)$ patients (BAMBOO) and three of $10(30 \%)$, three of 10 $(30 \%)$, and three of five ( $60 \%)$ patients (CYPRESS) had gained at least 15 letters in BCVA from baseline in the abicipar $1 \mathrm{mg}$, abicipar $2 \mathrm{mg}$, and ranibizumab arms, respectively.

\section{Anatomic Outcomes}

Improvements in mean change in CRT from baseline were observed in each treatment arm at all follow-up visits in both the BAMBOO and CYPRESS 


\begin{tabular}{|c|c|c|c|c|c|c|}
\hline \multicolumn{7}{|c|}{$\begin{array}{c}\text { TABLE } 3 \\
\text { Adverse Events Reported in Two or More Patients Across Studies, } \mathbf{n}(\%)\end{array}$} \\
\hline \multirow[b]{2}{*}{ Adverse Event } & \multicolumn{3}{|c|}{ BAMBOO (Japan) } & \multicolumn{3}{|c|}{ CYPRESS (United States) } \\
\hline & $\begin{array}{l}\text { Abicipar } 1 \mathrm{mg} \\
(\mathrm{n}=10)\end{array}$ & $\begin{array}{l}\text { Abicipar } 2 \mathrm{mg} \\
(\mathrm{n}=10)\end{array}$ & $\begin{array}{l}\text { RAN } 0.5 \mathrm{mg} \\
(\mathrm{n}=5)\end{array}$ & $\begin{array}{l}\text { Abicipar } 1 \mathrm{mg} \\
(\mathrm{n}=10)\end{array}$ & $\begin{array}{l}\text { Abicipar } 2 \mathrm{mg} \\
(\mathrm{n}=10)\end{array}$ & $\begin{array}{l}\text { RAN } 0.5 \mathrm{mg} \\
(\mathrm{n}=5)\end{array}$ \\
\hline $\begin{array}{l}\text { Conjunctival } \\
\text { hemorrhage }\end{array}$ & $4(40)$ & $0(0)$ & $0(0)$ & $0(0)$ & $0(0)$ & $1(20)$ \\
\hline Dry eye & $1(10)$ & $0(0)$ & $0(0)$ & $1(10)$ & $1(10)$ & $0(0)$ \\
\hline Cataract & $1(10)$ & $0(0)$ & $1(20)$ & $0(0)$ & $0(0)$ & $0(0)$ \\
\hline Eye pain & $0(0)$ & $0(0)$ & $0(0)$ & $0(0)$ & $1(10)$ & $1(20)$ \\
\hline $\begin{array}{l}\text { Foreign body } \\
\text { sensation in eyes }\end{array}$ & $0(0)$ & $1(10)$ & $1(20)$ & $0(0)$ & $0(0)$ & $0(0)$ \\
\hline $\begin{array}{l}\text { Intraocular pressure } \\
\text { increased }\end{array}$ & $0(0)$ & $0(0)$ & $2(40)$ & $0(0)$ & $0(0)$ & $0(0)$ \\
\hline Iritis & $1(10)$ & $1(10)$ & $0(0)$ & $0(0)$ & $0(0)$ & $0(0)$ \\
\hline Oedema peripheral & $0(0)$ & $0(0)$ & $0(0)$ & $0(0)$ & $1(10)$ & $1(20)$ \\
\hline Pneumonia & $0(0)$ & $0(0)$ & $1(20)$ & $0(0)$ & $1(10)$ & $0(0)$ \\
\hline Vitreous floaters & $1(10)$ & $1(10)$ & $0(0)$ & $0(0)$ & $0(0)$ & $0(0)$ \\
\hline Vitreous opacities & $1(10)$ & $1(10)$ & $0(0)$ & $0(0)$ & $0(0)$ & $0(0)$ \\
\hline Vitritis & $1(10)$ & $0(0)$ & $0(0)$ & $0(0)$ & $1(10)$ & $0(0)$ \\
\hline$R A N=$ ranibizumab & & & & & & \\
\hline
\end{tabular}

studies (Figure 4). Mean (SEM) change in CRT from baseline at week 16, 8 weeks after the last abicipar injection and 4 weeks after the last ranibizumab injection, was -187.3 (46.1) $\mu \mathrm{m},-196.5$ (39.3) $\mu \mathrm{m}$, and $-230.4(26.5) \mu \mathrm{m}$ in the BAMBOO study and -106.5 (40.6) $\mu \mathrm{m},-112.8$ (53.7) $\mu \mathrm{m}$, and -124.4 (22.1) $\mu \mathrm{m}$ in the CYPRESS study in the abicipar $1 \mathrm{mg}$, abicipar 2 $\mathrm{mg}$, and ranibizumab arms, respectively (Figure 4). At week 20, 12 weeks after the last abicipar injection and 4 weeks after the last ranibizumab injection, mean (SEM) change from baseline in CRT was -139.4 (35.0) $\mu \mathrm{m},-194.9$ (34.6) $\mu \mathrm{m}$, and $-250.6(22.7) \mu \mathrm{m}$ in the BAMBOO study and -78.2 (33.1) $\mu \mathrm{m},-90.3$ (56.4) $\mu \mathrm{m}$, and -110.4 (20.5) in the CYPRESS study in the abicipar $1 \mathrm{mg}$, abicipar $2 \mathrm{mg}$, and ranibizumab arms, respectively (Figure 4).

\section{Subgroup Analysis by Presence/Absence of PCV}

ICGA in the BAMBOO study demonstrated the presence of PCV in 14 of 25 patients (56\%). Subgroup analysis of efficacy endpoints in the BAMBOO study by the presence or absence of PCV showed that abicipar effectively improved BCVA and CRT in patients with PCV, as well as in patients without PCV (Table 2). Mean change from baseline in BCVA at week 16 was +6.0 letters, +9.8 letters, and +12.3 letters for patients with PCV and +9.6 letters, +7.5 letters, and +25.0 letters for patients without PCV in the abicipar $1 \mathrm{mg}$, abicipar $2 \mathrm{mg}$, and ranibizumab arms, respectively. Mean change from baseline in CRT at week 16 was $-268.6 \mu \mathrm{m},-191.2 \mu \mathrm{m}$, and $-253.7 \mu \mathrm{m}$ for patients with PCV and $-106.0 \mu \mathrm{m},-204.5 \mu \mathrm{m}$, and $-195.5 \mu \mathrm{m}$ for patients without PCV in the abicipar $1 \mathrm{mg}$, abicipar $2 \mathrm{mg}$, and ranibizumab arms, respectively.

\section{Case Studies}

Effects of abicipar treatment in two patients are illustrated in Figures 5 and 6. Figure 5 shows visual and anatomic outcomes in a 91-year-old Caucasian female who was treated with abicipar $2 \mathrm{mg}$ at day 1, week 4, and week 8 in the CYPRESS study. At baseline, BCVA in the study eye was 56 letters (approximately 20/80 Snellen equivalent). One week after the first intravitreal injection of abicipar, CRT was reduced and BCVA had improved by 17 letters. BCVA of 73 letters (approximately 20/32 Snellen equivalent) or better was sustained through week 20.

Visual and anatomic outcomes in a 77-year-old Japanese male who was treated with abicipar $2 \mathrm{mg}$ in the BAMBOO study are shown in Figure 6. Screening ICGA demonstrated a PCV component of the lesion 
TABLE 4

Visual Outcomes After Intraocular Inflammation AEs

\begin{tabular}{|c|c|c|c|c|c|}
\hline $\begin{array}{l}\text { Intraocular } \\
\text { Inflammation } \\
\text { Adverse Event } \\
\text { Preferred Term }\end{array}$ & Study & Treatment Arm & $\begin{array}{l}\text { Baseline BCVA } \\
\text { (Letters) }\end{array}$ & $\begin{array}{l}\text { Change From } \\
\text { Baseline BCVA } \\
\text { After AE (Letters) }\end{array}$ & $\begin{array}{l}\text { BCVA Change } \\
\text { From Baseline } \\
\text { at Study Exit } \\
\text { (Letters) }\end{array}$ \\
\hline $\begin{array}{l}\text { Anterior chamber } \\
\text { inflammation }\end{array}$ & BAMBOO & Abicipar 1 mg & 46 & +16 & +16 \\
\hline Iritis* & BAMBOO & Abicipar $1 \mathrm{mg}$ & 24 & +21 & +21 \\
\hline Vitritis* & BAMBOO & Abicipar $1 \mathrm{mg}$ & 24 & +21 & +21 \\
\hline Vitreous opacities & BAMBOO & Abicipar $1 \mathrm{mg}$ & 59 & +1 & +8 \\
\hline Iritis & BAMBOO & Abicipar 2 mg & 51 & 0 & +4 \\
\hline Vitreous opacities & BAMBOO & Abicipar 2 mg & 73 & +8 & +12 \\
\hline Iridocyclitis & CYPRESS & Abicipar 1 mg & 66 & -20 & +2 \\
\hline Uveitis & CYPRESS & Abicipar 2 mg & 73 & -38 & -2 \\
\hline Vitritis & CYPRESS & Abicipar 2 mg & 42 & -18 & +8 \\
\hline
\end{tabular}

in the study eye. At baseline, BCVA was 41 letters (approximately 20/160 Snellen equivalent). Abicipar treatment was administered at day 1 , week 4 , and week 8. By week 12, CRT was reduced and BCVA had improved by 20 letters. These improvements persisted through week 20, 12 weeks after the last abicipar injection. At week 20, BCVA was 63 letters (approximately 20/50).

\section{Safety Outcomes}

The overall incidence of AEs was eight of 10 $(80 \%)$, four of $10(40 \%)$, and five of five $(100 \%)$ in the BAMBOO study and five of 10 (50\%), six of 10 $(60 \%)$, and two of five ( $40 \%)$ in the CYPRESS study in the abicipar $1 \mathrm{mg}$, abicipar $2 \mathrm{mg}$, and ranibizumab arms, respectively. The most common AE was conjunctival hemorrhage (Table 3), which was associated with the injection procedure. All AEs that were considered possibly related to treatment were ocular, and none were serious. AEs of intraocular inflammation were reported in the abicipar arms; each of these AEs is listed in Table 4. These AEs were generally mild to moderate in severity, and all resolved with no treatment or with topical, intravitreal, or oral corticosteroid therapy. None of the AEs of intraocular inflammation were associated with a sustained loss of vision (Table 4). No AEs of intraocular inflammation were reported in the ranibizumab arms. Across the BAMBOO and CYPRESS studies, uveitis or vitritis was reported in three of 40 abicipar-treated patients compared with zero of 10 ranibizumab-treated patients.

All patients in the BAMBOO study (25 of 25 [100\%]) demonstrated stable vision throughout the study. In the CYPRESS study, 22 of 25 (88\%) patients had stable vision at all scheduled study visits. With respect to patients in the CYPRESS study who did not achieve stable vision, one patient in the abicipar $2 \mathrm{mg}$ arm had a 16-letter loss in BCVA from baseline on day 8 because of treatment-related vitritis; the vitritis resolved and the patient subsequently achieved an 8-letter BCVA gain from baseline. In addition, at week 20 , one patient in each abicipar treatment arm had a 22- or 30-letter loss in BCVA from baseline because of an AE unrelated to treatment (progression of RPE atrophy or central retinal vein occlusion, respectively).

There were no Antiplatelet Trialists' Collaboration (APTC) arterial thromboembolic events (ATEs) reported in the abicipar treatment arms of either BAMBOO or CYPRESS. In the ranibizumab arm in the BAMBOO study, however, there was an APTC ATE report of embolic stroke. There was also one report of increased blood pressure in the ranibizumab arm of the BAMBOO study. There were no reports of proteinuria, hemorrhagic events, or delayed woundhealing.

\section{DISCUSSION}

nAMD is characterized by a sudden vision loss, and anti-VEGF agents such as ranibizumab have 
changed clinical practice for the treatment of this disease. ${ }^{1}$ In registration studies of anti-VEGF treatment in $\mathrm{nAMD},{ }^{3,4,19}$ vision was improved or stabilized in the majority of patients, and the effects on vision were associated with significantly improved visionrelated quality-of-life outcomes. ${ }^{20,21}$ Consequently, intravitreal injections of anti-VEGF agents have become the foundation of therapy for nAMD. ${ }^{1}$ However, in order to maintain optimal efficacy, frequent intravitreal injections are required. In clinical studies, monthly injections of anti-VEGF therapies have provided the best efficacy outcomes. ${ }^{22}$ Thus there is an unmet medical need for a therapy that maintains maximal efficacy while requiring less frequent injections. Such a therapy would potentially remove a significant treatment burden on patients and their health care providers, and also decrease the risk of complications associated with intravitreal injections such as endophthalmitis, retinal detachment, and elevated intraocular pressure. The BAMBOO and CYPRESS studies evaluated abicipar safety and efficacy in treatment-naïve patients with nAMD. The studies demonstrated comparable abicipar safety and a duration of abicipar efficacy of up to 12 weeks after the last dose in both Japanese and non-Japanese patients with nAMD. The results of the BAMBOO study further suggest that abicipar is effective in treating Japanese patients with PCV.

Patients in the abicipar treatment arms of the BAMBOO and CYPRESS studies received abicipar $1 \mathrm{mg}$ or $2 \mathrm{mg}$. These abicipar doses were the same as those evaluated in stage 3 of the phase 2 REACH study. Across all three studies, abicipar $2 \mathrm{mg}$ demonstrated a greater, more consistent treatment effect than abicipar $1 \mathrm{mg}$, supporting the use of abicipar $2 \mathrm{mg}$ in ongoing phase 3 trials of abicipar for treatment of nAMD (clinicaltrials.gov NCT02462486, NCT02462928). The BAMBOO and CYPRESS data suggest that abicipar effects on BCVA are durable and maintained at least 12 weeks after three monthly injections, confirming previous results in the REACH stage 3 study. Overall, the phase 2 data suggest that abicipar could provide an extended duration of effect and efficacy similar to that demonstrated by ranibizumab in its pivotal studies, but with reduced burden of visits for patients and health care providers.

At week 16, 8 weeks after the last abicipar injection, abicipar $2 \mathrm{mg}$ provided mean gains in BCVA of +8.9 and +10.1 letters in the BAMBOO and CYPRESS studies, respectively. These effects are consistent with those observed in REACH stage 3, where abicipar $2 \mathrm{mg}$ achieved a mean gain in BCVA of 8.2 letters at week $16 .{ }^{16}$ In addition, overall gains in these three studies are consistent with BCVA improvements at week 16 after monthly injections of ranibizumab 0.5 $\mathrm{mg}$ in the MARINA and ANCHOR registration trials of ranibizumab for treatment of nAMD (+9.9 letters in $\mathrm{ANCHOR}^{3}$ and approximately +6.1 letters in MARINA (estimation from digitization using Engauge Digitizer software [available at: http://engauge-digitizer. soft112.com] of MARINA study data presented in Figure 2A of Rosenfeld et al. ${ }^{4}$ ) Evaluation of response to ranibizumab in the BAMBOO and CYPRESS studies indicates that patients (five in each study) responded unexpectedly well to ranibizumab, with week 16 mean BCVA gains of +17.4 letters and +15.2 letters, respectively. The reason for this response, which is almost double that reported in ANCHOR, MARINA, and REACH stage 3, is difficult to understand. The most likely explanation is the small number of patients randomized to the active control arm (ie, by randomization chance these arms included a high percentage of patients who were highly responsive to ranibizumab).

With respect to vision stabilization, abicipar stabilized vision (no loss in BCVA $\geq 15$ letters) in almost all patients treated with abicipar in the BAMBOO and CYPRESS studies. These data are also consistent with those reported in the REACH stage 3 study in which all patients treated with abicipar at either dose ( 1 or 2 $\mathrm{mg}$ ) achieved stable vision. ${ }^{16}$

Abicipar induced similar improvements in anatomical outcomes in BAMBOO and CYPRESS, highlighting the comparability of the Japanese and United States study populations in their CRT response to abicipar. The improvements were observed in each treatment arm at all follow-up visits for both studies. Since the last dose of abicipar was administered at week 8, maintenance of effect in anatomical outcomes reflects an extended duration of action of abicipar. Mean reduction in CRT from baseline was $187.3 \mu \mathrm{m}$, $196.5 \mu \mathrm{m}$, and $230.4 \mu \mathrm{m}$ in the BAMBOO study and $106.5 \mu \mathrm{m}, 112.8 \mu \mathrm{m}$, and $124.4 \mu \mathrm{m}$ in the CYPRESS study at week 16, and $139.4 \mu \mathrm{m}, 194.9 \mu \mathrm{m}$, and 250.6 $\mu \mathrm{m}$ in the BAMBOO study and $78.2 \mu \mathrm{m}, 90.3 \mu \mathrm{m}$, and $110.4 \mu \mathrm{m}$ in the CYPRESS study at week 20, in the abicipar $1 \mathrm{mg}$, abicipar $2 \mathrm{mg}$, and ranibizumab arms, respectively. The CRT changes observed in the BAMBOO and CYPRESS studies are similar to those in the REACH stage 3 study, in which the mean CRT reductions from baseline were $161 \mu \mathrm{m}, 111 \mu \mathrm{m}$, and $127 \mu \mathrm{m}$ at week 16 and $147 \mu \mathrm{m}, 102 \mu \mathrm{m}$, and $125 \mu \mathrm{m}$ at week 20 in the abicipar $1 \mathrm{mg}$, abicipar $2 \mathrm{mg}$, and ranibizumab $0.5 \mathrm{mg}$ arms, respectively. ${ }^{16}$ These data support comparable effects of abicipar on anatomical endpoints in Japanese and non-Japanese patients and also provide evidence of a long duration of action of abicipar not only on functional endpoints (BCVA) as 
described above, but also on retinal anatomy (CRT), with effects that can be observed at least 12 weeks after the last abicipar administration.

The effects of anti-VEGF treatment in patients with nAMD may be influenced by the subtype of nAMD. ${ }^{23,24}$ PCV is a distinct form of neovascular AMD that is characterized by a network of branching choroidal vessels with terminal, polyp-like dilations that can be visualized on ICGA. It is frequently associated with serosanguineous detachments of the RPE. PCV and typical nAMD differ in epidemiological and clinical characteristics, natural history, and treatment outcomes. ${ }^{9}$ It has been suggested that the neovascular lesions in typical AMD and PCV may respond differently to anti-VEGF therapy because of differences in the mechanisms underlying the neovascularization..$^{25}$ Although ranibizumab, bevacizumab, and aflibercept have all demonstrated efficacy in improving vision, decreasing foveal thickness, and causing polyp regression in patients with PCV, ${ }^{26-28}$ ranibizumab therapy may be more effective in eyes with typical nAMD than in eyes with PCV. ${ }^{8}$ In the BAMBOO study, abicipar had beneficial effects on BCVA and CRT in both patients with PCV and patients with typical nAMD.

The safety profile of abicipar demonstrated in the BAMBOO and CYPRESS studies was acceptable. There were no AEs potentially related to systemic VEGF inhibition in the abicipar treatment arms. All treatment-related AEs in the abicipar arms were ocular, and none were serious. The most common AE was conjunctival hemorrhage associated with the injection procedure.

The BAMBOO and CYPRESS studies included treatment-naïve patients to allow a more robust evaluation of the treatment effects of abicipar and to prevent confounding effects from any previous treatment. Study strengths included the use of ICGA and subgroup analysis based on the presence or absence of PCV in the Japanese patient population. Study limitations included the small sample sizes and the 6-month study duration. A sustained duration of effect of abicipar relative to ranibizumab was inferred from the efficacy demonstrated by abicipar at 8 and 12 weeks after the last of three monthly injections. We acknowledge that a study design that included a treatment arm with three monthly ranibizumab injections, perhaps followed by a treat-and-extend option, would have allowed a more direct comparison of the duration of treatment effects of abicipar and ranibizumab. However, because this study was conducted to fulfil regulatory agency requirements, monthly administration of ranibizumab per its approved drug labelling was required. The effects of extended treatment with abicipar $2 \mathrm{mg}$ at 8 -week or 12-week intervals are under investigation in ongoing phase 3 studies.

In summary, abicipar performed similarly in Japanese and non-Japanese patients in the BAMBOO and CYPRESS studies, despite the presence of PCV in more than half of the Japanese patient population. Functional improvements, anatomic effects, and safety were comparable between abicipar $1 \mathrm{mg}$ and $2 \mathrm{mg}$ treatment arms in each study. Improvements in BCVA and CRT with abicipar were sustained through week 20, 12 weeks after the last abicipar injection, demonstrating a long duration of abicipar effects. Improvements in BCVA and CRT were similar in PCV and non-PCV Japanese patients receiving abicipar $2 \mathrm{mg}$. Overall, the BAMBOO and CYPRESS data on abicipar confirm those from REACH stage 3. The data indicate that abicipar could achieve functional and anatomical improvements in nAMD, but with a reduced number of injections and dosing intervals of 8 weeks or 12 weeks, consistent with greater duration of action. The results of the phase 2 studies support further clinical development of abicipar $2 \mathrm{mg}$ for treatment of nAMD.

\section{REFERENCES}

1. Yonekawa Y, Kim IK. Clinical characteristics and current treatment of age-related macular degeneration. Cold Spring Harb Perspect Med. 2014;5(1):a017178.

2. Schmidt-Erfurth U, Chong V, Loewenstein A, et al. Guidelines for the management of neovascular age-related macular degeneration by the European Society of Retina Specialists (EURETINA). Br J Ophthalmol. 2014;98(9):1144-1167.

3. Brown DM, Kaiser PK, Michels M, et al. Ranibizumab versus verteporfin for neovascular age-related macular degeneration. $N$ Engl J Med. 2006;355(14):1432-1444.

4. Rosenfeld PJ, Brown DM, Heier JS, et al. Ranibizumab for neovascular age-related macular degeneration. $N$ Engl J Med. 2006;355(14):14191431.

5. Holz FG, Amoaku W, Donate J, et al. Safety and efficacy of a flexible dosing regimen of ranibizumab in neovascular age-related macular degeneration: The SUSTAIN study. Ophthalmology. 2011;118(4):663671

6. Singer MA, Awh CC, Sadda S, et al. HORIZON: An open-label extension trial of ranibizumab for choroidal neovascularization secondary to age-related macular degeneration. Ophthalmology. 2012;119(6):1175-1183.

7. Kim JH, Lee TG, Chang YS, Kim CG, Cho SW. Short-term choroidal thickness changes in patients treated with either ranibizumab or aflibercept: A comparative study. $\mathrm{Br} J$ Ophthalmol. 2016;100(12):1634-1639.

8. Matsumiya W, Honda S, Otsuka K, et al. Comparison of the effectiveness and prognostic factors of intravitreal ranibizumab between typical neovascular age-related macular degeneration and polypoidal choroidal vasculopathy over 24 months of follow-up. Ophthalmologica. 2015;234(1):33-39.

9. Wong CW, Yanagi Y, Lee WK, et al. Age-related macular degeneration and polypoidal choroidal vasculopathy in Asians. Prog Retin Eye Res. 2016;53:107-139.

10. Ozkaya A, Alagoz C, Garip R, et al. The role of indocyanine green angiography imaging in further differential diagnosis of patients with 
nAMD who are morphologically poor responders to ranibizumab in a real-life setting. Eye (Lond). 2016;30(7):958-965.

11. Campochiaro PA, Channa R, Berger BB, et al. Treatment of diabetic macular edema with a designed ankyrin repeat protein that binds vascular endothelial growth factor: A phase I/II study. Am J Ophthalmol. 2013;155(4):697-704.e1-2.

12. Souied EH, Devin F, Mauget-Faÿsse M, et al; MP0112 Study Group. Treatment of exudative age-related macular degeneration with a designed ankyrin repeat protein that binds vascular endothelial growth factor: A phase I/II study. Am J Ophthalmol. 2014;158(4):724-732.e2.

13. Smithwick E, Stewart MW. Designed ankyrin repeat proteins: A look at their evolving use in medicine with a focus on the treatment of chorioretinal vascular disorders. Antiinflamm Antiallergy Agents Med Chem. 2017;16(1):33-45.

14. Papadopoulos N, Martin J, Ruan Q, et al. Binding and neutralization of vascular endothelial growth factor (VEGF) and related ligands by VEGF Trap, ranibizumab and bevacizumab. Angiogenesis. 2012;15(2):171-185.

15. Krohne TU, Liu Z, Holz FG, Meyer CH. Intraocular pharmacokinetics of ranibizumab following a single intravitreal injection in humans. Am J Ophthalmol. 2012;154(4):682-686.e2.

16. Callanan D, Kunimoto D, Maturi RK, et al. Double-masked, randomized, phase 2 evaluation of abicipar pegol (an anti-VEGF DARPin therapeutic) in neovascular age-related macular degeneration. $J$ Ocul Pharmacol Ther. 2018;34(10):700-709.

17. Maruko I, Iida T, Saito M, Nagayama D, Saito K. Clinical characteristics of exudative age-related macular degeneration in Japanese patients. Am J Ophthalmol. 2007;144(1):15-22.e2.

18. Age-Related Eye Disease Study Research Group. The Age-Related Eye Disease Study (AREDS): Design implications. AREDS report no. 1. Control Clin Trials. 1999;20(6):573-600.

19. Heier JS, Brown DM, Chong V, et al. Intravitreal aflibercept (VEGF trap-eye) in wet age-related macular degeneration. Ophthalmology. 2012;119(12):2537-2548.

20. Chang TS, Bressler NM, Fine JT, Dolan CM, Ward J, Klesert TR. Improved vision-related function after ranibizumab treatment of neovascular age-related macular degeneration: Results of a randomized clinical trial. Arch Ophthalmol. 2007;125(11):1460-1469.

21. Bressler NM, Chang TS, Suñer IJ, et al. Vision-related function after ranibizumab treatment by better- or worse-seeing eye: clinical trial results from MARINA and ANCHOR. Ophthalmology. 2010;117(4):747-756.e4.

22. Holekamp NM, Liu Y, Yeh WS, et al. Clinical utilization of antiVEGF agents and disease monitoring in neovascular age-related macular degeneration. Am J Ophthalmol. 2014;157(4):825-833.e1.

23. Hirakata T, Fujinami K, Watanabe K, Sasaki M, Noda T, Akiyama $\mathrm{K}$. One-year outcome of intravitreal aflibercept injection for age-related macular degeneration resistant to ranibizumab: Rapid morphologic recovery and subsequent visual improvement. Clin Ophthalmol. 2016;10:969-977.

24. Kim JH, Chang YS, Lee DW, Kim CG, Kim JW. Incidence and timing of the first recurrence in neovascular age-related macular degeneration: Comparison between ranibizumab and aflibercept. J Ocul Pharmacol Ther. 2017;33(6):445-451.

25. Balaratnasingam C, Dhrami-Gavazi E, McCann JT, Ghadiali Q, Freund KB. Aflibercept: A review of its use in the treatment of choroidal neovascularization due to age-related macular degeneration. Clin Ophthalmol. 2015;9:2355-2371.

26. Hikichi T. Individualized ranibizumab therapy strategies in year 3 after as-needed treatment for polypoidal choroidal vasculopathy. $B M C$ Ophthalmol. 2015;15:37.

27. Cho HJ, Baek JS, Lee DW, Kim CG, Kim JW. Short-term effectiveness of intravitreal bevacizumab vs. ranibizumab injections for patients with polypoidal choroidal vasculopathy. Korean J Ophthalmol. 2012;26(3):157-162.

28. Cho HJ, Kim KM, Kim HS, et al. Intravitreal aflibercept and ranibizumab injections for polypoidal choroidal vasculopathy. Am J Ophthalmol. 2016;165:1-6. 